\title{
Multimodal prognosis of negative symptom severity in individuals at increased risk of developing psychosis
}

Daniel J. Hauke $\mathbb{B}^{1,2}$, André Schmidt $\mathbb{D}^{1}$, Erich Studerus ${ }^{3}$, Christina Andreou ${ }^{1,4}$, Anita Riecher-Rössler (D)', Joaquim Radua ${ }^{5,6,7}$, Joseph Kambeitz $\mathbb{1 0}^{8}$, Anne Ruef ${ }^{9}$, Dominic B. Dwyer ${ }^{9}$, Lana Kambeitz-llankovic ${ }^{8,9}$, Theresa Lichtenstein ${ }^{8}$, Rachele Sanfelici ${ }^{9,10}$, Nora Penzel ${ }^{8,9}$, Shalaila S. Haas (1) ${ }^{11}$, Linda A. Antonucci ${ }^{12}$, Paris Alexandros Lalousis (10 ${ }^{13}$, Katharine Chisholm ${ }^{14}$, Frauke Schultze-Lutter (15,16,17, Stephan Ruhrmann (1)

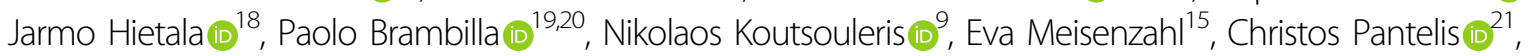
Marlene Rosen ${ }^{8}$, Raimo K. R. Salokangas ${ }^{18}$, Rachel Upthegrove $\mathbb{E}^{22}$, Stephen J. Wood ${ }^{14,23,24}$ and Stefan Borgwardt $\mathbb{B}^{1,4}$, and the PRONIA Group

\begin{abstract}
Negative symptoms occur frequently in individuals at clinical high risk (CHR) for psychosis and contribute to functional impairments. The aim of this study was to predict negative symptom severity in CHR after 9 months. Predictive models either included baseline negative symptoms measured with the Structured Interview for Psychosis-Risk Syndromes (SIPS-N), whole-brain gyrification, or both to forecast negative symptoms of at least moderate severity in $94 \mathrm{CHR}$. We also conducted sequential risk stratification to stratify CHR into different risk groups based on the SIPS-N and gyrification model. Additionally, we assessed the models' ability to predict functional outcomes in CHR and their transdiagnostic generalizability to predict negative symptoms in 96 patients with recent-onset psychosis (ROP) and 97 patients with recent-onset depression (ROD). Baseline SIPS-N and gyrification predicted moderate/severe negative symptoms with significant balanced accuracies of 68 and $62 \%$, while the combined model achieved $73 \%$ accuracy. Sequential risk stratification stratified CHR into a high (83\%), medium (40-64\%), and low (19\%) risk group regarding their risk of having moderate/severe negative symptoms at 9 months follow-up. The baseline SIPS-N model was also able to predict social (61\%), but not role functioning (59\%) at above-chance accuracies, whereas the gyrification model achieved significant accuracies in predicting both social (76\%) and role (74\%) functioning in CHR. Finally, only the baseline SIPS-N model showed transdiagnostic generalization to ROP (63\%). This study delivers a multimodal prognostic model to identify those CHR with a clinically relevant negative symptom severity and functional impairments, potentially requiring further therapeutic consideration.
\end{abstract}

Correspondence: Daniel J. Hauke (daniel.hauke@unibas.ch)

${ }^{1}$ Department of Psychiatry (UPK), University of Basel, Basel, Switzerland ${ }^{2}$ Department of Mathematics and Computer Science, University of Basel, Basel, Switzerland

Full list of author information is available at the end of the article

The authors contributed equally: Daniel J. Hauke, André Schmidt.

PRONIA Group: A full list of members and their affiliations appears in the

Supplementary Information.

\section{Introduction}

Precise prognosis of clinical outcomes in individuals at clinical high risk (CHR) of developing psychosis is imperative to guide treatment selection. Prognostic risk stratification models help to decide who will benefit most from active treatment ${ }^{1}$. While much effort has been dedicated to predicting transition to psychosis ${ }^{2,3}$, prognostic models focusing on negative symptom outcomes in

\section{(c) The Author(s) 2021}

(c) (i) Open Access This article is licensed under a Creative Commons Attribution 4.0 International License, which permits use, sharing, adaptation, distribution and reproduction c. in any medium or format, as long as you give appropriate credit to the original author(s) and the source, provide a link to the Creative Commons license, and indicate if changes were made. The images or other third party material in this article are included in the article's Creative Commons license, unless indicated otherwise in a credit line to the material. If material is not included in the article's Creative Commons license and your intended use is not permitted by statutory regulation or exceeds the permitted use, you will need to obtain permission directly from the copyright holder. To view a copy of this license, visit http://creativecommons.org/licenses/by/4.0/. 
CHR are missing. This is a major oversight, bearing in mind that $82 \%$ of $\mathrm{CHR}$ exhibit at least one negative symptom in the moderate to severe range at first clinical presentation, and $54 \%$ still meet this criterion after 12 months $^{4}$. Negative symptoms are also strong predictors of poor functioning ${ }^{5,6}$ irrespective of other symptoms such as depression or anxiety ${ }^{7}$. Prognostic tools are therefore urgently required to track negative symptom progression in CHR and to identify those who might benefit most from potential interventions, such as $N$-methyl-D-asparate-receptor (NMDAR) modulators (in conjunction with psychosocial interventions ${ }^{8}$ ).

A key challenge in management of psychotic disorders is that clinical outcomes are difficult to prognosticate based on behavioural signs ${ }^{9}$. To overcome this issue, the field is searching for biologically informed prognostic assays ${ }^{10}$ to complement clinical information for increased treatment precision and/or prognostic indication ${ }^{11,12}$. Sequentially adding biological evidence to initial clinical assessments may help establish more accurate stratification models to forecast clinical outcomes in $\mathrm{CHR}^{13,14}$. Considering psychosis as a brain disorder ${ }^{1,15}$, neuroimaging offers a powerful tool to map pathophysiological processes associated with illness onset. However, most neuroimaging studies to date have reported differences at the group level, rendering personalized clinical decisionmaking difficult. Machine-learning provides a promising tool to address this issue ${ }^{16,17}$. For instance, a recent machine-learning study in CHR found that grey matter volume and clinical data could predict individual social functioning with more than $75 \%$ accuracy, while a combination of models further improved prognostic performance to $82 \%{ }^{18}$. Furthermore, using surface-based measures like gyrification and surface area, other CHR studies showed that transition to psychosis or global functioning can be predicted with more than $80 \%$ accuracy $^{19,20}$. The measurement of gyrification might be particularly sensitive to detect pathophysiology in the prodromal phase ${ }^{21}$. Gyrification is critical during early brain maturation ${ }^{22,23}$. Early maldevelopment may result in an intransient risk factor for emerging psychosis. This aligns with the developmental risk model of psychosis ${ }^{24}$, which integrates perinatal hazards and neurodevelopmental abnormalities with stressful experiences during adolescence into the pathogenesis of psychosis.

Here, we applied machine-learning ${ }^{3,17,25}$ to multisite data ${ }^{18}$ to predict negative symptoms of clinically relevant severity after 9 months using baseline negative symptoms measured with the Structured Interview for PsychosisRisk Syndromes ${ }^{26}$ (SIPS-N), gyrification data, and their combination as predictors. Second, we conducted a twostep simulation approach ${ }^{13,14}$ by sequentially adding the gyrification model on top of the baseline SIPS-N model to stratify individuals into different risk groups. Third, bearing in mind that negative symptoms were related to poor functioning in $\mathrm{CHR}^{5,6}$, we assessed the ability of the three negative symptom models to predict functional impairments. Finally, we investigated transdiagnostic generalizability by employing the models trained in CHR to predict negative symptom severity in recent-onset psychosis (ROP) and recent-onset depression (ROD) patients. This last analysis is grounded in evidence showing an overlap between negative and the depressive symptoms ${ }^{27}$, pluripotent, transdiagnostic trajectories of CHR including non-psychotic disorders ${ }^{11,28}$, and riskassociated disruption of brain circuits that may mediate susceptibility to broad domains of psychopathology rather than discrete disorders ${ }^{29}$. This holistic strategy is useful for predicting clinical outcomes transdiagnostically, paving the way for the development of transdiagnostic, preventative interventions.

\section{Subjects and methods \\ Subjects}

As part of a European multisite study, the 'Personalized Prognostic Tools for Early Psychosis Management' (PRONIA; http://www.pronia.eu; see ref. ${ }^{18}$ for more details on project design), CHR, ROP, and ROD patients were recruited at seven sites in five countries. Only individuals with complete negative symptom and gyrification data at baseline and negative symptom assessment at the 9-month assessment were included yielding 94 CHR, 96 ROP, and 97 ROD. Written informed consent was obtained from all participants. The authors assert that all procedures contributing to this work comply with the ethical standards of the relevant national and institutional committees on human experimentation and with the Helsinki Declaration of 1975, as revised in 2008. All procedures involving human participants were approved by the respective local ethics committee.

General inclusion criteria were age between 15 and 40 years, sufficient language skills for participation, as well as capacity to provide informed consent. General exclusion criteria were an IQ below 70, current or past head trauma with loss of consciousness $(>5 \mathrm{~min}$ ), current or past known neurological or somatic disorders, current or past alcohol dependence, or polysubstance dependence within the past 6 months, cannabis consumption in the last month and any medical indication against MRI. The CHR state was defined by either (a) cognitive disturbances (COGDIS) criteria assessed using the Schizophrenia Proneness Instrument (SPI-A) ${ }^{30}$ (see refs. ${ }^{31,32}$ for more details) and/or (b) ultra-high-risk criteria for psychosis based on the Structured Interview for Psychosis-Risk Syndromes $\left(\right.$ SIPS $\left.^{26}\right)$ (see refs. ${ }^{32,33}$ for more details). Exclusion criteria for CHR were (i) antipsychotic medication for $>30$ days (cumulative number of days) at or above minimum dosage of the '1st episode psychosis' 
range of DGPPN S3 guidelines ${ }^{34}$ and (ii) any intake of antipsychotic medication within the past 3 months before clinical baseline assessments at or above minimum dosage of the '1st episode psychosis' range of DGPPN S3 guidelines $^{34}$. Detailed in- and exclusion criteria for the ROP and ROD groups can be found in the supplement.

\section{Clinical assessment}

All participants underwent clinical assessment of positive, negative, disorganized, and general symptoms using the SIPS $^{26}$. Furthermore, depressive symptoms were assessed with Beck's Depression Inventory (BDI) ${ }^{35}$, and social and role functioning with the Global Functioning: Social and Role scales ${ }^{36}$.

\section{Neuroimaging assessment}

Gyrification was derived from a T1-weighted structural MRI. (See Table S1 for data acquisition parameters). All images were processed and analysed using the Computational Anatomy Toolbox (CAT12; version r1155; http:// dbm.neuro.uni-jena.de/cat12/), an extension toolbox of Statistical Parametric Mapping software (SPM12, http:// www.fil.ion.ucl.ac.uk/spm/software/spm12). Gyrification values were then extracted for 68 regions (34 for each hemisphere) using the Desikan-Killiany atlas ${ }^{37}$ as implemented in CAT12 and normalized for individual intracranial volumes given that gyrification may correlate with the brain volume ${ }^{38}$. More detailed information is provided in the Supplement.

\section{Outcome definition}

\section{Negative symptom outcomes}

According to Cornblatt and colleagues, ${ }^{49-41}$, individuals with a score of $\geq 3$ in any of the SIPS negative symptom items can be considered as having moderate to severe negative symptoms. Scores equal to or greater than 3 on the SIPS have been rated as clinically significant ${ }^{42}$ and $54 \%$ of CHR still exhibit such symptoms after 12 months ${ }^{4}$. Notably, SIPS negative symptom items are more severe and persistent in individuals who convert to psychosis $^{4}$ and longer duration and severity of SIPS negative symptom items are related to poor social functioning ${ }^{41}$. The outcome label was defined using N1 (social anhedonia), N2 (avolition), N3 (expression of emotion), N4 (experience of emotions and self) and N6 (occupational functioning); we excluded N5 (ideational richness) because several factor analyses found N5 to be either unrelated to the other negative symptoms ${ }^{43,44}$ or only weakly related to them when they were exclusively examined ${ }^{45}$. The latter factor analysis further indicated a two-factor model with two SIPS negative symptom dimensions reflecting volition (avolition and occupational functioning) and emotion (expression and experience of emotion and social anhedonia) ${ }^{45}$. While the volition factors showed an association with poor role function, the emotion factor was associated with poor social function.

\section{Functional outcomes}

In line with a recent analysis ${ }^{18}$, we used the Global Functioning: Social and Role scales ${ }^{36}$ to define adequate (more than 7 points) vs impaired (7 or fewer points) social and role functioning at a 9-month follow-up assessment.

\section{Predictor variables \\ Baseline SIPS-N model}

The baseline SIPS- $\mathrm{N}$ model used individual SIPS baseline items $\mathrm{N} 1, \mathrm{~N} 2, \mathrm{~N} 3, \mathrm{~N} 4$, and $\mathrm{N} 6$ as predictors.

\section{Gyrification model}

The gyrification model used individual gyrification values for all 68 regions as predictors.

\section{Statistical analysis \\ Machine-learning analysis to predict negative symptom severity}

We trained three different models to predict negative symptom outcomes in CHR using the NeuroMiner software package (version: 1.0; release: elessar; https://www. pronia.eu/neurominer) in Matlab (release: R2017a; https://de.mathworks.com/products/matlab.html). The first two models were trained using baseline SIPS-N and gyrification data as predictors, respectively (see above). The third model constituted a stacking model that combined predictions of the SIPS- $\mathrm{N}$ and gyrification model. Methodological details of model construction can be found in the supplement. We used nested cross-validation with a leave-site-out cross-validation (LSO-CV) in the outer loop to assess geographic generalizability to a hypothetical new centre and fivefold repeated crossvalidation with 10 repetitions in the inner loop. To assess whether models achieved above-chance performance, we computed the posterior distributions of the balanced accuracy (BAC) $)^{46}$ and considered the performance to be significant, if $95 \%$ of the posterior mass fell above a BAC of 0.5 , as 0.5 indicates chance level in a binary classification context. Note that we report the LSO-CV point estimate, but also computed other moments of the posterior distribution and positive and negative predictive values $(\mathrm{PPV} / \mathrm{NPV}))$. To further investigate which predictors reliably drove the classification performance of the models, we computed cross-validation ratio profiles $(\mathrm{CVR}=\operatorname{mean}(w) / \mathrm{SE}(w)$, where $w$ corresponds to the normalized weight vector under Euclidian assumptions of the linear classifier (see ref. ${ }^{18}$ for more details).

\section{Sequential risk stratification}

In real-world clinical settings clinical information is usually collected sequentially to minimize the burden for 
patients and healthcare costs, rather than simultaneously as in our study. To simulate this scenario, we performed a sequential risk stratification based on the base rate of experiencing moderate/severe negative symptoms and the sensitivity and specificity of the baseline SIPS-N and gyrification model derived from our sample. The goal of this analysis was to stratify $\mathrm{CHR}$ into different risk groups to guide future treatment selection by estimating the theoretical PPV of a two-stage probabilistic assessment approach (see refs. ${ }^{13,14}$ for technical details). In brief, starting with the pretest probability of having moderate/ severe negative symptoms at follow-up in our own CHR sample $(40 \%)$, we simulated a hypothetical scenario in which each individual would be subjected first to a clinical baseline assessment using the SIPS negative items and subsequently undergo a second test, here a structural MRI, from which a gyrification signature would be computed. Following this procedure, we were able to stratify individuals into different risk groups with respect to the probability of presenting moderate/severe negative symptoms at follow-up. Based on the risk ratio of a recent clinical pilot trial in $\mathrm{CHR}^{47}$, we finally assessed the theoretical clinical efficacy of this two-stage sequential testing approach by estimating the number needed to treat (NNT) for each risk group to achieve remission of negative symptoms when undergoing a D-serine treatment (for a schematic analysis overview see Fig. S1).

\section{Assessing outcome generalization of negative symptom models to functional outcomes in CHR}

Next, we assessed whether the models that were trained on baseline data to predict negative symptoms in CHR were also able to predict adequate vs impaired social and role functioning at follow-up in the same group.

\section{Assessing transdiagnostic generalization of negative symp- tom models to other patient populations}

To test transdiagnostic generalizability of models, we applied the negative symptom models trained in the CHR sample to patients with ROP and ROD.

\section{Results and discussion \\ Results}

\section{Demographic and clinical features}

Demographics and clinical characteristic of the whole study sample are described in Table 1, S2, and S3. In the CHR group, 38 individuals (40\%) presented at least one negative symptom rated $\geq 3$ on the SIPS (i.e. moderate/ severe severity) at follow-up, while 56 patients $(60 \%)$ displayed mild or no negative symptoms (scores $\leq 2$ ). The groups did not differ in age, sex, handedness, and education. The moderate/severe negative symptom group showed higher negative symptoms and lower social/role functioning at baseline and follow-up (see Fig. S2). To assess whether negative symptom outcomes were confounded by secondary negative symptoms ${ }^{48}$, we computed correlations between outcomes, and SIPS positive as well as BDI depressive symptoms at baseline. This analysis suggested that negative symptoms at follow-up could not be explained by secondary negative symptoms resulting from positive $(r=-0.069, p=1.000)$ or depressive symptoms $(r=-0.075, p=0.941)$.

In the ROP group, 57 patients (59\%) showed moderate/ severe negative symptom outcomes, whereas 39 patients (41\%) exhibited mild negative symptoms. These groups did not differ in age, handedness, but in sex and education. ROP patients with moderate/severe negative symptom outcomes at follow-up presented higher negative symptoms and lower social/role functioning at baseline and follow-up.

In the ROD group, 31 patients (32\%) suffered from moderate/severe negative symptoms at follow-up, while 66 patients $(68 \%)$ showed mild negative symptoms. These two groups did not differ in age, sex, handedness, and education. Similar to the other groups moderate/severe negative symptom outcomes were associated with higher negative symptoms and poorer social, but not role functioning at baseline and higher negative symptoms as well as poorer social/role functioning at follow-up.

\section{Multimodal prognosis of negative symptom severity in $\mathrm{CHR}$ patients}

Baseline SIPS-N model The baseline SIPS-N model achieved an above-chance BAC of $68 \%$ in predicting moderate/severe negative symptoms at follow-up in CHR (CI: [62\%, 81\%]; Fig. 1A and Table 2). Feature ranking showed that social anhedonia contributed most robustly to the classification, followed by occupational functioning and avolition (Fig. 2A).

Gyrification model The gyrification model achieved a significant BAC of $62 \%$ (CI: [52\%, 71\%]; Fig. $1 \mathrm{~A}$ and Table 2). Feature ranking showed that reduced gyrification in the left pericalcarine gyrus, left posterior superior temporal sulcus (pSTS), left supramarginal gyrus, right transverse temporal gyrus, and increased gyrification in the left inferior frontal gyrus (IFG) (pars triangularis) was associated with increased risk of expressing moderate/ severe negative symptoms contributing most to the classification (Fig. 2B). Gyrification values for each region and group are reported in Table S4.

Combined model Combining the baseline SIPS-N and gyrification model improved the BAC to $73 \%$ (CI: [63\%, 81\%]; Fig. 1A and Table 2).

Sequential risk stratification Sequentially adding prognostic performance of the gyrification model on top of the 


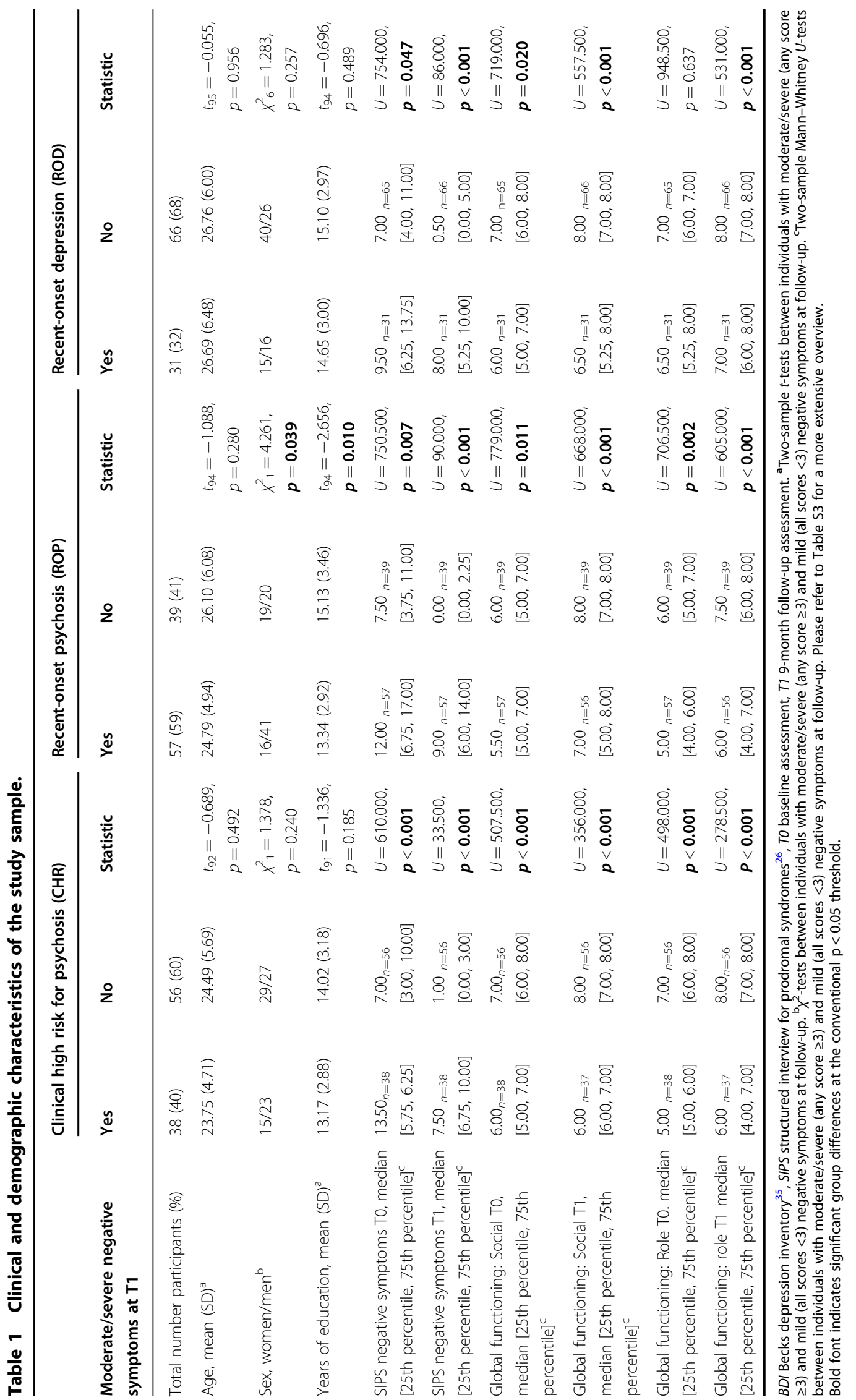


A
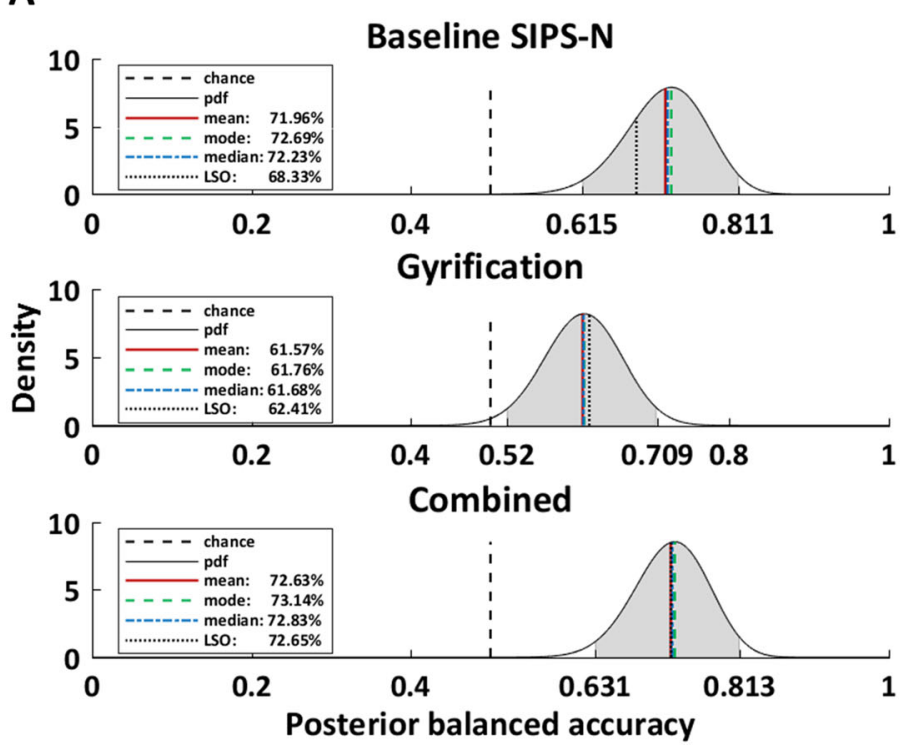

B
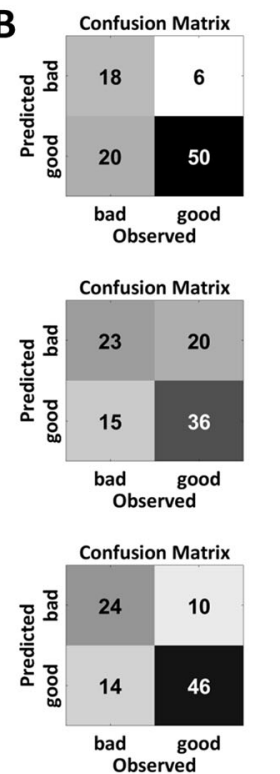
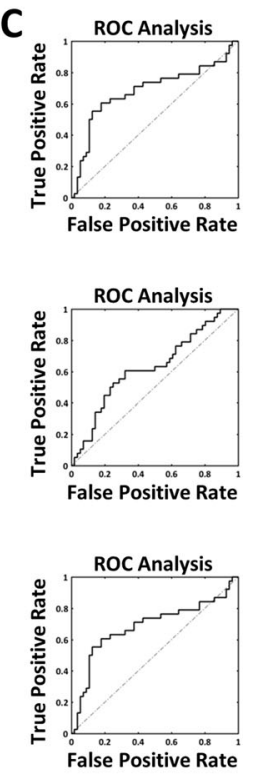

Fig. 1 Model performances. A Posterior balanced accuracy distributions of baseline negative symptom (upper panel), gyrification (middle panel), and combined model (lower panel). Shaded grey area indicates $95 \%$ of the probability mass of the respective posterior distribution over the balanced accuracy. B Confusion matrices and $\mathbf{C}$ receiver operating characteristic (ROC) curves of the prediction models. Bad outcome: Expression of moderate to severe negative symptoms at follow-up (any score $\geq 3$ ). Good outcome: All SIPS negative items $<3$ at follow-up. SIPS-N negative symptoms measured the Structured Interview for Psychosis-Risk Syndromes ${ }^{26}$.

baseline SIPS-N model led to a PPV of 83\% (empirical probability in our sample: $91 \%$ ) for an individual with two positive tests (high-risk group), 64 and 40\% (empirical probabilities in our sample: 62 and 41\%) for an individual with one positive and one negative test (medium-risk group) and 19\% (empirical probabilities in our sample: $18 \%$ ) for an individual with no positive tests (low-risk group; Fig. 2C). Accordingly, the hypothetical NNT for a D-serine intervention was 1 for those with two positive tests, 2-3 for those with one positive test, and 6 for those with no positive test (see Fig. 2C).

Outcome generalization of negative symptom models to functional outcomes in CHR Social and role functioning data of one participant were missing. For the remaining $93 \mathrm{CHR}$, the baseline SIPS-N model produced an above-chance BAC for social $(62 \%$; $\mathrm{CI}$ : [52\%, 72\%], see Fig. S3A and Table 2), but not role functioning (59\%; CI: [50\%, 70\%]). The gyrification model generalized to both role (74\%; CI: [64\%, $81 \%]$ ) and social functioning (76\%; CI: [67\%, 83\%]) with very high accuracy. Lastly, the combined model also predicted role (63\%; CI: [54\%, 73\%]) and social functioning (64\%; CI: [56\%, 74\%]) with intermediate BACs.

Transdiagnostic generalization of negative symptom models to other patient populations The baseline SIPS$\mathrm{N}$ model generalized to ROP (63\%; CI: [53\%, 71\%], see Fig.
S3B and Table 2), but not to ROD patients (57\%; CI: [48\%, $70 \%])$. In contrast, the gyrification model failed to generalize to both ROP (55\%; CI: [45\%, 65\%]) and ROD patients (48\%; CI: [39\%, 57\%]). Lastly, the combined model generalized to ROP (64\%; CI: [54\%, 72\%]), but not ROD patients (60\%; CI: [50\%, 68\%]).

\section{Discussion}

To the best of our knowledge this is the first study using state-of-the-art predictive modelling ${ }^{49}$ to forecast negative symptom severity in CHR after 9 months. Four major results were obtained: First, SIPS-N and gyrification baseline data predicted the presentation of moderate/ severe negative symptom expression with an abovechance BAC of $68 \%$ and $62 \%$, respectively, while the combined model achieved 73\% BAC. Secondly, sequential testing allowed the stratification of CHR individuals into high (83\%), medium (40-64\%), and low (19\%) groups regarding their risk to present moderate to severe negative symptoms at follow-up. Thirdly, we found that the SIPS$\mathrm{N}$ model was also able to predict social (61\%), but not role functioning at above-chance accuracy in CHR, whereas the gyrification model predicted both role $(74 \%)$ and social $(76 \%)$ functioning with high accuracies. The combined model also predicted role (63\%) and social (65\%) functioning. Finally, we found that the SIPS-N and the combined $(63 \%, 64 \%)$, but not the gyrification model 
Table 2 Classification performance, outcome generalization performance and transdiagnostic generalization performance.

\begin{tabular}{|c|c|c|c|c|c|c|}
\hline & LSO-BAC (\%) & AUC (\%) & SE (\%) & SP (\%) & PPV (\%) & NPV (\%) \\
\hline \multicolumn{7}{|c|}{ Classification performance of prognostic models } \\
\hline Baseline SIPS-N model & $68^{*}[62,81]$ & 68 & 47 & 89 & 75 & 71 \\
\hline Gyrification model & $62 *[52,71]$ & 63 & 61 & 64 & 53 & 71 \\
\hline Combined model & $73^{*}[63,81]$ & 71 & 63 & 82 & 71 & 63 \\
\hline \multicolumn{7}{|c|}{ Generalization to GF R outcome } \\
\hline Baseline SIPS-N model & $59[50,70]$ & 67 & 37 & 85 & 76 & 52 \\
\hline Gyrification model & $74^{*}[64,81]$ & 79 & 65 & 83 & 83 & 65 \\
\hline Combined model & $63^{*}[53,72]$ & 69 & 48 & 78 & 74 & 54 \\
\hline \multicolumn{7}{|c|}{ Generalization to GF S outcome } \\
\hline Baseline SIPS-N model & $61 *[52,72]$ & 67 & 37 & 85 & 76 & 51 \\
\hline Gyrification model & $76^{*}[67,83]$ & 80 & 67 & 85 & 85 & 67 \\
\hline Combined model & $65^{*}[56,74]$ & 70 & 50 & 80 & 76 & 56 \\
\hline \multicolumn{7}{|l|}{ Generalization to ROP } \\
\hline Baseline SIPS-N model & $63^{*}[53,71]$ & 63 & 51 & 74 & 74 & 51 \\
\hline Gyrification model & $55[45,65]$ & 53 & 35 & 74 & 67 & 44 \\
\hline Combined model & $64 *[54,72]$ & 63 & 67 & 62 & 72 & 56 \\
\hline \multicolumn{7}{|l|}{ Generalization to ROD } \\
\hline Baseline SIPS-N model & $57[48,70]$ & 62 & 32 & 82 & 45 & 72 \\
\hline Gyrification model & $48[39,57]$ & 49 & 45 & 50 & 30 & 66 \\
\hline Combined model & $60[50,68]$ & 61 & 55 & 65 & 43 & 75 \\
\hline
\end{tabular}

SIPS- $N$ negative symptoms measured with the structured interview for prodromal syndromes ${ }^{26}, L S O-B A C$ leave-site-out balanced accuracy point estimate, $A U C$ area under the curve, SE sensitivity, $S P$ specificity, $P P V$ positive predictive value, NPV negative predictive value, GF $R$ global functioning role outcome ${ }^{36}$, GF $S$ global functioning social outcome ${ }^{36}$. Square brackets indicate lower and upper limit of $95 \%$ confidence interval around posterior balanced accuracy estimate. indicates significantly better than chance level (i.e., $50 \%$ not included in $95 \%$ of the probability mass of the posterior distribution over the model's balanced accuracy). Bold font indicates signficantly better than chance level (i.e.,50\% not included in $95 \%$ of the probability mass of the posterior distribution over the model's balanced accuracy).

generalized to ROP, but not ROD patients for negative symptom prediction.

\section{Baseline expression of negative symptoms predicts negative symptom severity at follow-up}

We found that baseline expression of negative symptoms predicted moderate/severe negative symptoms in CHR with 68\% BAC. Social anhedonia contributed most to the prognostic performance, followed by occupational functioning and avolition. This finding resonates with recent results showing that premorbid social maladjustment in late adolescence strongly predicted social anhedonia, and to a lesser extent occupational functioning, avolition, and expression of emotions in $\mathrm{CHR}^{50}$. It has been argued that a reciprocal relationship may exist between premorbid social adjustment and social anhedonia, possibly aggravating negative symptoms over time $^{51}$. Our baseline SIPS-N model also achieved abovechance BAC in predicting impaired social functioning in $\mathrm{CHR}$, generally supporting the inverse relationship between negative symptoms and social functioning in $\mathrm{CHR}^{5}$. It did not, however, generalize to role functioning. A recent factor analysis demonstrated that SIPS negative symptoms reflect two factors: (1) volition including avolition and occupational functioning and (2) emotion subsuming expression of emotion, social anhedonia, and experience of emotion ${ }^{45}$. The authors further reported that the emotion factor was associated with poor social function, whereas the volition factor was more related to poor role function ${ }^{45}$. Since social anhedonia (emotion factor) was weighted strongest in our model, it may be expected that the model generalizes better to social rather than role functioning. Lastly, we observed that the baseline SIPS-N model partially generalized to ROP patients, which may be explained by the large phenomenological overlap of the two populations. Interestingly, the baseline SIPS-N model did not generalize to ROD patients in our sample, possibly indicating specificity to the psychosis spectrum. This result resonates with evidence suggesting that at least some negative symptoms (e.g. social 


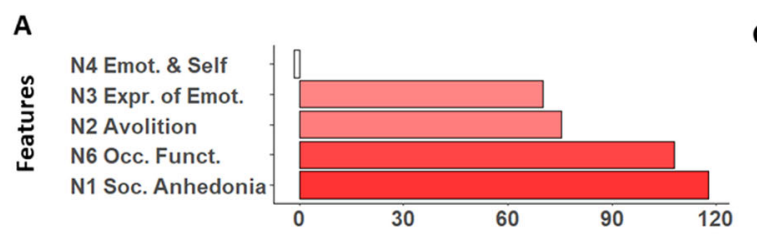

B
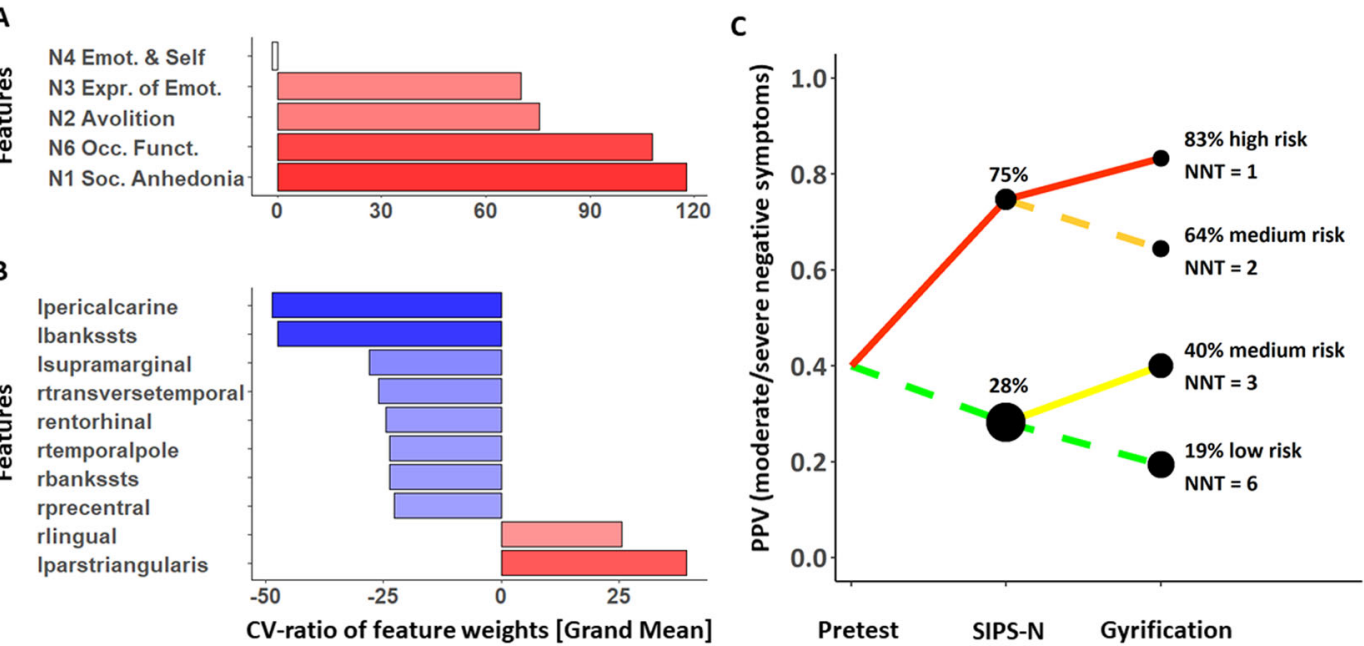

Fig. 2 Feature importance. A Feature importance of baseline negative symptom model. Feature importance was measured through crossvalidation $(\mathrm{CV})$ ratio profiles $(\mathrm{CVR}=$ mean $(\mathrm{W}) / \mathrm{SE}(\mathrm{W})$, where $w$ corresponds to the normalized weight vector under Euclidian assumptions of the logistic regression or support vector machine classifier; see ref. ${ }^{18}$ for more details). Negative CVRs indicate that reduced values of the predictor are associated with increased risk of expressing negative symptoms, whereas positive values imply that an increase of the predictor value is associated with increased risk. B Top 10 most important features of the gyrification model. C Probabilistic assessment diagram illustrating two-stage sequential risk stratification to stratify patients based on their risk to develop moderate to severe negative symptoms. Note that this computation is based on (1) the base rate, as well as sensitivity and specificity from (2) the baseline SIPS-N and (3) the gyrification model, all derived from our sample. $X$-axis: Sequential tests (based on baseline negative symptoms and gyrification). Y-axis: Positive predictive value (PPV) associated with expressing moderate/ severe negative symptoms at 9 months follow-up. The pretest probability was set to $40 \%$ based on our own sample (see Table 1). Each bifurcation in the plot represents the updated PPV after knowing that a test either yielded a positive (ascending solid line) or a negative result (descending dashed line). For a schematic analysis overview, please refer to Fig. S1. Line colour: Level of risk as previously suggested ${ }^{14}$. High: $>80 \%$, medium: 40-64\%, and low: $<20 \%$. Dot sizes: Relative proportion of participants in our sample with a corresponding number of positive tests. The diagram also illustrates the number needed to treat (NNT) at each node, which is based on the risk ratio of a recent clinical pilot trial with a D-serine intervention to treat negative symptoms in clinical high-risk individuals ${ }^{47}$. SIPS-N negative symptoms measured the Structured Interview for Psychosis-Risk Syndromes ${ }^{26}$.

anhedonia) may be temporally linked to depressive episodes, whereas they appear to be more trait-like in the psychosis spectrum ${ }^{52,53}$. Another recent study found an association of the volition factor with subsyndromal depressive symptoms in bipolar I disorder, but not in schizophrenia ${ }^{54}$, highlighting the possibility that negative symptoms of similar severity may indeed be caused by distinct mechanisms in these two disorders.

\section{Baseline gyrification predicts negative symptom severity at follow-up}

Secondly, we found that whole-brain gyrification pattern also predicted moderate/severe negative symptoms in CHR with $62 \%$ BAC. In particular, we found that left pericalcarine gyrus, pSTS, left pars triangularis/IFG, left supramarginal gyrus, and right transverse temporal gyrus contributed most to the classification. In $80 \%$ of the top ten predictors, reduced gyrification was associated with increased risk of expressing moderate /severe negative symptoms, which is in line with previous evidence indicating that overall gyrification reductions are related to the severity of negative symptoms in patients with schizophrenia $^{55}$. A number of these regions pertain to the 'mentalizing network' ${ }^{56}$. Specifically, the pSTS has been implicated in perception of biological motion ${ }^{57}$, and gaze tracking ${ }^{58}$, but also, in conjunction with the temporoparietal junction, in implicit mentalizing ${ }^{59}$. The IFG has also been related to this network ${ }^{60,61}$, as mirror neurons have been identified there ${ }^{62}$ and lesions in this region lead to deficits in emotional empathy ${ }^{63}$. Predominance of these regions in our gyrification model may also explain why we found strong generalization performances to social and role functioning outcomes. Furthermore, the gyrification model did not generalize to ROD patients. These results resonate with other findings showing that the overlap changes in cortical gyrification in patients with schizophrenia and bipolar disorder is small $(25 \%)^{64}$, and that there may be distinct mechanisms underlying negative symptoms in depression vs psychosis spectrum disorders, as alluded to above. Interestingly, the gyrification model did also not generalize to ROP, possibly indicating that developmental changes in gyrification provide a unique signature for the psychosis high-risk state.

Simultaneous and sequential multimodal prediction of negative symptom severity at follow-up

We observed a prognostic benefit when combining the SIPS-N and gyrification model, leading to a BAC of $73 \%$. 
Furthermore, sequentially adding the prognostic performance of the gyrification model on top of the baseline SIPS-N model allowed us to stratify CHR patients into high (83\%), medium (40-64\%), and low (19\%) group, regarding their risk to present moderate/severe negative symptoms after 9 months. These three risk groups also exhibited different responder characteristics to potential interventions. In particular, using data from a previous Dserine trial in CHR patients aiming to treat negative symptoms ${ }^{47}$, we showed that the high-risk group would most benefit from such an intervention $(\mathrm{NNT}=1)$, the medium-risk group at an intermediate level $(\mathrm{NNT}=2-3)$, and the low-risk group least $(\mathrm{NNT}=6)($ Fig. $2 \mathrm{C})$. This suggests that the assessment of gyrification as a potential complement to initial clinical assessments may be valuable for patient stratification, for example, to assign patients to different treatment arms ${ }^{13}$ in clinical trials.

\section{Limitations}

Some limitations of our study merit comment. Remission of negative symptoms in CHR has been defined as a $20 \%$ improvement based on pilot study after D-serine treatment ${ }^{47}$. However, remission of negative symptoms has not been conceptualized yet as categorical outcome and bearing in mind the small size in this study, sequential testing findings should be interpreted with caution. Furthermore, although our models were constructed in a comparatively large CHR sample, they need to be validated in an independent dataset, which may also help to arbitrate between using the SIPS-N or the combined model. For some applications the clinical model alone may already be sufficient to achieve a relevant degree of stratification, which can be integrated easily in care routines, but is associated with a larger degree of uncertainty. For other applications, our models may need to be enriched with more clinical and biological predictors to improve prognosis. The field will have to discuss what accuracy is required for clinical implementation. This should be considered in light of down-stream consequences of being assigned to a specific risk group (for example, increasing monitoring rate, may be ethically justifiable with worse performance compared to administration of medication with potentially serious side effects).

\section{Conclusions}

In conclusion, this study provides a multimodal prognostic model for negative symptoms severity and functional impairments in CHR. Using clinical and gyrification data, we deliver a pragmatic strategy to identify those CHR individuals with clinically relevant negative symptom severity and functional impairments, potentially requiring specialized care. Such multimodal and multistep prognostic testing may help to stratify individual risk profiles and optimize personalized interventions in the future.

\section{Acknowledgements}

We gratefully acknowledge the participants for their contributions. This work has been presented as an oral contribution at the 2020 Congress of the Schizophrenia International Research Society.

\section{Author details}

${ }^{1}$ Department of Psychiatry (UPK), University of Basel, Basel, Switzerland. ${ }^{2}$ Department of Mathematics and Computer Science, University of Basel, Basel, Switzerland. ${ }^{3}$ Department of Psychology, University of Basel, Basel, Switzerland. ${ }^{4}$ Department of Psychiatry and Psychotherapy, University of Lübeck, Lübeck, Germany. ${ }^{5}$ Imaging of Mood- and Anxiety-Related Disorders (IMARD) Group, Institut d'Investigacions Biomèdiques August Pi i Sunyer (IDIBAPS), CIBERSAM, Barcelona, Spain. ${ }^{6}$ Early Psychosis: Interventions and Clinical-Detection (EPIC) Lab, Department of Psychosis Studies, Institute of Psychiatry, Psychology and Neuroscience, King's College London, London, UK. ${ }^{7}$ Department of Clinical Neuroscience, Karolinska Institute, Stockholm, Sweden. ${ }^{8}$ Department of Psychiatry and Psychotherapy, Faculty of Medicine and University Hospital, University of Cologne, Cologne, Germany. ${ }^{9}$ Department of Psychiatry and Psychotherapy, Ludwig-Maximilian-University, Munich, Germany. ${ }^{10}$ Max Planck School of Cognition, Leipzig, Germany. ${ }^{11}$ Department of Psychiatry, Icahn School of Medicine at Mount Sinai, New York, NY, USA. ${ }^{12}$ Department of Education, Psychology, Communication, University of Bari Aldo Moro, Bari, Italy. ${ }^{13}$ Institute for Mental Health and Centre for Human Brain Health, University of Birmingham, Birmingham, UK. ${ }^{14}$ Institute for Mental Health, University of Birmingham, Birmingham, UK. ${ }^{15}$ Department of Psychiatry and Psychotherapy, Medical Faculty, Heinrich-Heine University, Düsseldorf, Germany. ${ }^{16}$ University Hospital of Child and Adolescent Psychiatry and Psychotherapy, University of Bern, Bern, Switzerland. ${ }^{17}$ Department of Psychology and Mental Health, Faculty of Psychology, Airlangga University, Surabaya, Indonesia. ${ }^{18}$ Department of Psychiatry, University of Turku, Turku, Finland. ${ }^{19}$ Department of Neurosciences and Mental Health, Fondazione IRCCS Ca' Granda Ospedale Maggiore Policlinico, Milan, Italy. ${ }^{20}$ Department of Pathophysiology and Transplantation, University of Milan, Milan, Italy. ${ }^{21}$ Melbourne Neuropsychiatry Centre, University of Melbourne \& Melbourne Health, Carlton South, VIC, Australia. ${ }^{22}$ Institute for Mental Health and School of Psychology, University of Birmingham, Birmingham, UK. ${ }^{23}$ Orygen, Melbourne, VIC, Australia. ${ }^{24}$ Centre for Youth Mental Health, University of Melbourne, Melbourne, VIC, Australia

\section{Code availability}

Analysis code will be provided upon reasonable request to the corresponding author.

\section{Conflict of interest}

This work was funded by the European Union under the 7th Framework Programme (grant number 602152). D.J.H.'s work was supported by the Swiss National Science Foundation (Ambizione grant; grant number 167952). J.R.'s work was supported by Miguel Servet Research Contract (CPII19/00009) and Research Projects PI19/00394 from the Plan Nacional de I + D + i, the Instituto de Salud Carlos III-Subdirección General de Evaluación y Fomento de la Investigación and the European Regional Development Fund (FEDER, 'Investing in your future'). R.S. received honoraria for one lecture from Lundbeck outside the submitted work and funding from BMBF and the Max Planck Society. C.P. has received honoraria for talks at educational meetings and has served on an advisory board for Lundbeck, Australia Pty Ltd, and was supported by a National Health and Medical Research Council (NHMRC) Senior Principal Research Fellowship (1105825), an NHMRC L3 Investigator Grant (1196508), and NHMRC-EU Grant (ID: 1075379). The remaining authors declare no competing interests.

\section{Publisher's note}

Springer Nature remains neutral with regard to jurisdictional claims in published maps and institutional affiliations.

Supplementary information The online version contains supplementary material available at https://doi.org/10.1038/s41398-021-01409-4. 
Received: 13 October 2020 Revised: 12 April 2021 Accepted: 30 April 2021 Published online: 24 May 2021

\section{References}

1. Insel, T. R. \& Cuthbert, B. N. Medicine. Brain disorders? Precisely. Science $\mathbf{3 4 8}$ 499-500 (2015).

2. Riecher-Rössler, A. \& Studerus, E. Prediction of conversion to psychosis in individuals with an at-risk mental state: a brief update on recent developments. Curr. Opin. Psychiatry 30, 209-219 (2017).

3. Studerus, E., Ramyead, A. \& Riecher-Rössler, A. Prediction of transition to psychosis in patients with a clinical high risk for psychosis: a systematic review of methodology and reporting. Psychol. Med. 47, 1163-1178 (2017).

4. Piskulic, D. et al. Negative symptoms in individuals at clinical high risk of psychosis. Psychiatry Res. 196, 220-224 (2012).

5. Meyer, E. C. et al. The relationship of neurocognition and negative symptoms to social and role functioning over time in individuals at clinical high risk in the first phase of the North American Prodrome Longitudinal Study. Schizophr. Bull. 40, 1452-1461 (2014).

6. Lee, S. J., Kim, K. R., Lee, S. Y. \& An, S. K. Impaired social and role function in ultra-high risk for psychosis and first-episode schizophrenia: its relations with negative symptoms. Psychiatry Invest. 14, 539-545 (2017).

7. Schlosser, D. A. et al. Modeling the role of negative symptoms in determining social functioning in individuals at clinical high risk of psychosis. Schizophr. Res. 169, 204-208 (2015).

8. Devoe, D. J., Peterson, A. \& Addington, J. Negative symptom interventions in youth at risk of psychosis: a systematic review and network meta-analysis. Schizophr. Bull. 44, 807-823 (2018).

9. McGuire, P. \& Dazzan, P. Does neuroimaging have a role in predicting outcomes in psychosis? World Psychiatry 16, 209-210 (2017).

10. Singh, I. \& Rose, N. Biomarkers in psychiatry. Nature 460, 202-207 (2009).

11. McGorry, P. D., Hartmann, J. A., Spooner, R. \& Nelson, B. Beyond the "at risk mental state" concept: transitioning to transdiagnostic psychiatry. World Psychiatry 17, 133-142 (2018).

12. Schultze-Lutter, F., Schmidt, S. J. \& Theodoridou, A. Psychopathology-a precision tool in need of re-sharpening. Front. Psychiatry 9, 446 (2018).

13. Schmidt, A. et al. Improving prognostic accuracy in subjects at clinical high risk for psychosis: systematic review of predictive models and meta-analytical sequential testing simulation. Schizophr. Bull. 43, 375-388 (2017).

14. Clark, S. R., Schubert, K. O. \& Baune, B. T. Towards indicated prevention of psychosis: using probabilistic assessments of transition risk in psychosis prodrome. J. Neural Transm. Nienna) 122, 155-169 (2015).

15. Insel, T. R. \& Quirion, R. Psychiatry as a clinical neuroscience discipline. JAMA 294, 2221-2224 (2005).

16. Bzdok, D. \& Meyer-Lindenberg, A. Machine learning for precision psychiatry: opportunities and challenges. Biol. Psychiatry Cogn. Neurosci. Neuroimaging 3, 223-230 (2018).

17. Janssen, R. J., Mourão-Miranda, J. \& Schnack, H. G. Making individual prognoses in psychiatry using neuroimaging and machine learning. Biol. Psychiatry Cogn. Neurosci. Neuroimaging 3, 798-808 (2018).

18. Koutsouleris, N. et al. Prediction models of functional outcomes for individuals in the clinical high-risk state for psychosis or with recent-onset depression: a multimodal, multisite machine learning analysis. JAMA Psychiatry 75, 1156-1172 (2018).

19. Das, T. et al. Disorganized gyrification network properties during the transition to psychosis. JAMA Psychiatry 75, 613-622 (2018).

20. Kambeitz-llankovic, L. et al. Prediction of outcome in the psychosis prodrome using neuroanatomical pattern classification. Schizophr. Res. 173, 159-165 (2016).

21. Palaniyappan, L. Progressive cortical reorganisation: a framework for investigating structural changes in schizophrenia. Neurosci. Biobehav. Rev. 79, 1-13 (2017).

22. Raznahan, A. et al. How does your cortex grow? J. Neurosci. 31, 7174-7177 (2011).

23. Raznahan, A. et al. Patterns of coordinated anatomical change in human cortical development: a longitudinal neuroimaging study of maturational coupling. Neuron 72, 873-884 (2011).

24. Murray, R. M., Bhavsar, V., Tripoli, G. \& Howes, O. 30 years on: how the neurodevelopmental hypothesis of schizophrenia morphed into the developmental risk factor model of psychosis. Schizophr. Bull. 43, 1190-1196 (2017).
25. Dwyer, D. B., Falkai, P. \& Koutsouleris, N. Machine learning approaches for clinical psychology and psychiatry. Annu. Rev. Clin. Psychol. 14, 91-118 (2018).

26. McGlashan, T., Walsh, B. \& Woods, S. The Psychosis-Risk Syndrome: Handbook for Diagnosis and Follow-up (Oxford University Press, 2010).

27. Krynicki, C. R., Upthegrove, R., Deakin, J. F. W. \& Barnes, T. R. E. The relationship between negative symptoms and depression in schizophrenia: a systematic review. Acta Psychiatr. Scand. 137, 380-390 (2018).

28. McGorry, P. \& Nelson, B. Why we need a transdiagnostic staging approach to emerging psychopathology, early diagnosis, and treatment. JAMA Psychiatry 73, 191-192 (2016).

29. Buckholtz, J. W. \& Meyer-Lindenberg, A. Psychopathology and the human connectome: toward a transdiagnostic model of risk for mental illness. Neuron 74, 990-1004 (2012).

30. Schultze-Lutter, F., Addington, J., Ruhrmann, S. \& Klosterkötter, J. Schizophrenia Proneness Instrument —Adult Version (Giovanni Fioriti Editore, 2007).

31. Schultze-Lutter, F., Klosterkötter, J. \& Ruhrmann, S. Improving the clinical prediction of psychosis by combining ultra-high risk criteria and cognitive basic symptoms. Schizophr. Res. 154, 100-106 (2014).

32. Schultze-Lutter, F. et al. EPA guidance on the early detection of clinical high risk states of psychoses. Eur. Psychiatry 30, 405-416 (2015).

33. Fusar-Poli, P. et al. The psychosis high-risk state: a comprehensive state-of-theart review. JAMA Psychiatry 70, 107-120 (2013).

34. Gaebel, W., Falkai, P., Weinmann, S. \& Wobrock, T. Praxisleitlinien in Psychiatrie und Psychotherapie, Band 1, Behandlungsleitlinie Schizophrenie (Deutsche Gesellschaft für Psychiatrie, Psychotherapie und Nervenheilkunde (DGPPN), 2006).

35. Beck, A. T., Ward, C. H., Mendelson, M., Mock, J. \& Erbaugh, J. An inventory for measuring depression. Arch. Gen. Psychiatry 4, 561-571 (1961).

36. Cornblatt, B. A. et al. Preliminary findings for two new measures of social and role functioning in the prodromal phase of schizophrenia. Schizophr. Bull. 33, 688-702 (2007).

37. Desikan, R. S. et al. An automated labeling system for subdividing the human cerebral cortex on MRI scans into gyral based regions of interest. Neuroimage 31, 968-980 (2006).

38. Toro, R. et al. Brain size and folding of the human cerebral cortex. Cereb. Cortex 18, 2352-2357 (2008).

39. Cornblatt, B. A. et al. The schizophrenia prodrome revisited: a neurodevelopmental perspective. Schizophr. Bull. 29, 633-651 (2003).

40. Lencz, T., Smith, C. W., Auther, A., Correll, C. U. \& Cornblatt, B. Nonspecific and attenuated negative symptoms in patients at clinical high-risk for schizophrenia. Schizophr. Res. 68, 37-48 (2004).

41. Carrión, R. E. et al. Duration of attenuated positive and negative symptoms in individuals at clinical high risk: associations with risk of conversion to psychosis and functional outcome. J. Psychiatr. Res. 81, 95-101 (2016).

42. Devoe, D. et al. Negative symptoms in youth at clinical high risk of psychosis. Schizophr. Bull. 43, S207 (2017).

43. Jung, M. H. et al. The reliability and validity of the korean version of the structured interview for prodromal syndrome. Psychiatry Investig. 7, 257-263 (2010).

44. Comparelli, A. et al. Factor-structure of the Italian version of the Scale Of Prodromal Symptoms (SOPS): a comparison with the English version. Epidemiol. Psychiatr. Sci. 20, 45-54 (2011).

45. Azis, M. et al. Factor analysis of negative symptom items in the structured interview for prodromal syndromes. Schizophr. Bull. 45, 1042-1050 (2019).

46. Brodersen, K. H., Ong, C. S., Stephan, K. E. \& Buhmann, J. M. The balanced accuracy and its posterior distribution. Proceedings of the 2010 20th International Conference on Pattern Recognition (IEEE, 2010).

47. Kantrowitz, J. T. et al. D-serine for the treatment of negative symptoms in individuals at clinical high risk of schizophrenia: a pilot, double-blind, placebocontrolled, randomised parallel group mechanistic proof-of-concept trial. Lancet Psychiatry 2, 403-412 (2015).

48. Kirschner, M., Aleman, A. \& Kaiser, S. Secondary negative symptoms - a review of mechanisms, assessment and treatment. Schizophr. Res. 186, 29-38 (2017).

49. Fusar-Poli, P., Hijazi, Z., Stahl, D. \& Steyerberg, E. W. The science of prognosis in psychiatry: a review. JAMA Psychiatry 75, 1289-1297 (2018).

50. Tarbox-Berry, S. I., Perkins, D. O., Woods, S. W. \& Addington, J. Premorbid social adjustment and association with attenuated psychotic symptoms in clinical high-risk and help-seeking youth. Psychol. Med. 48, 983-997 (2018).

51. Barkus, E. \& Badcock, J. C. A transdiagnostic perspective on social anhedonia. Front. Psychiatry 10, 216 (2019). 
52. Katsanis, J., lacono, W. G., Beiser, M. \& Lacey, L. Clinical correlates of anhedonia and perceptual aberration in first-episode patients with schizophrenia and affective disorder. J. Abnorm. Psychol. 101, 184-191 (1992).

53. Blanchard, J. J., Horan, W. P. \& Brown, S. A. Diagnostic differences in social anhedonia: a longitudinal study of schizophrenia and major depressive disorder. J. Abnorm. Psychol. 110, 363-371 (2001).

54. Kirschner, M. et al. Shared and dissociable features of apathy and reward system dysfunction in bipolar I disorder and schizophrenia. Psychol. Med. 50, 936-947 (2020).

55. Sallet, P. C. et al. Reduced cortical folding in schizophrenia: an MRI morphometric study. Am. J. Psychiatry 160, 1606-1613 (2003).

56. Frith, U. \& Frith, C. D. Development and neurophysiology of mentalizing. Philos. Trans. R. Soc. Lond. B Biol. Sci. 358, 459-473 (2003).

57. Puce, A. \& Perrett, D. Electrophysiology and brain imaging of biological motion. Philos. Trans. R. Soc. Lond. B Biol. Sci. 358, 435-445 (2003).

58. Hoffman, E. A. \& Haxby, J. V. Distinct representations of eye gaze and identity in the distributed human neural system for face perception. Nat. Neurosci. 3, 80-84 (2000).
59. Frith, C. D. \& Frith, U. Mechanisms of social cognition. Annu. Rev. Psychol. 63 287-313 (2012).

60. Mar, R. A. The neural bases of social cognition and story comprehension. Annu. Rev. Psychol. 62, 103-134 (2011).

61. Molenberghs, P., Johnson, H., Henry, J. D. \& Mattingley, J. B. Understanding the minds of others: a neuroimaging meta-analysis. Neurosci. Biobehav. Rev. 65, 276-291 (2016).

62. lacoboni, M. \& Dapretto, M. The mirror neuron system and the consequences of its dysfunction. Nat. Rev. Neurosci. 7, 942-951 (2006).

63. Shamay-Tsoory, S. G., Aharon-Peretz, J. \& Perry, D. Two systems for empathy: a double dissociation between emotional and cognitive empathy in inferior frontal gyrus versus ventromedial prefrontal lesions. Brain 132, 617-627 (2009).

64. Palaniyappan, L. \& Liddle, P. F. Diagnostic discontinuity in psychosis: a combined study of cortical gyrification and functional connectivity. Schizophr. Bull. 40, 675-684 (2014) 